\title{
Energy Efficient Designs of Sustainable Buildings in Urban Environment.
}

\author{
Doaa M.El-Sherif', Essam E. Khalil ${ }^{2}$, \\ ${ }^{1}$ Professor at HBRC, Cairo and \\ ${ }^{2}$ Fellow ASME, Fellow ASHRAE, Fellow AIAA, Professor of Mechanical Engineering, Cairo University-Egypt
}

\begin{abstract}
The Developing communities in their path for rapid development is endeavoring to make all necessary and appropriate measures to enhance the efficiency of energy utilization and increase the beneficiation of the energy resources. The energy production, transmission, distribution and utilization efficiency becomes a vital factor and measure of national development. Governmental organizations were established earlier to be responsible for energy planning and efficient utilization, information dissemination and capacity building as well as devising the necessary codes and standards. Throughout the Nation Energy resources are widely used and consumption rates are in general exceeding the International accepted values. Energy rationalization and audit exercises were developed and monitored by Governmental authorities, Universities and Research centers through the past two decades with a definitive positive energy reduction and beneficiation. The development of the relevant codes for Residential and Commercial Energy Efficiency in Building is underway through the governmental bodies responsible for the research, training and development in the building Technology sector and is the umbrella under which the National and Unified Arab Codes are developed and issued.
\end{abstract}

\section{Introduction}

As the world becomes increasingly dependent on electrical appliances and equipment, energy consumption rapidly rises every year. Many programs have been established in various countries to increase end-use equipment energy efficiency. One of the most costeffective and proven methods for increasing energy efficiency of electrical appliances and equipment is to establish energy efficiency standards and labels .Energy efficiency standards are a set of procedures and regulations that prescribe the energy performance of manufactured products, sometimes prohibiting the sale of products less energy-efficient than the minimum standard. The term "standard" commonly encompasses two possible meanings:

1. a well-defined protocol (or laboratory test procedure) by which to obtain a sufficiently accurate estimate of the energy performance of a product in the way it is typically used, or at least a relative ranking of the energy performance compared to other models; and

2. A target limit on energy performance (usually a maximum use or minimum efficiency) formally established by a government-based agency upon a specified test standard.
Energy efficiency labels are informative labels affixed to manufactured products indicating a product's energy performance (usually in the form of energy use, efficiency, and/or cost) in order to provide consumers with the data necessary for making informed purchases. Energy labels serve as a complement to energy standards. They provide consumers information that allows those who care to select more efficient models. Labels also allow utility companies and government energy conservation agencies to offer incentive $\mathrm{s}$ to consumers to buy the most energy-efficient products. The effectiveness of energy labels is highly dependent on how information is presented to the consumer.

\section{Literature Review and Background}

Energy efficiency in developed and developing countries plays an important role in achieving global sustainable development. Energy consumption is growing rapidly in these countries, yet energy efficiency remains far below levels in developed countries. Energy-efficiency improvements can slow the growth in energy consumption, save consumers money and reduce capital expenses for energy infrastructure, see references 1-15. For most developing countries, the foreign exchange needed to finance energy sector expansion is a significant drain on reserves. Additionally, energy efficiency reduces

\footnotetext{
Corresponding author: khalile1@asme.org
} 
local environmental impacts, such as water and air pollution from power plants, and mitigates greenhouse gas emissions. Standards and labeling programs provide enormous energy savings potential that can direct developing countries towards sustainable energy use. Improved end-use efficiency from Standards and Labeling programs can contribute significantly to developing economies. The main benefits are:

1. Less need to build new power plants. The cost of saving $1 \mathrm{kWh}$ of energy through energy-efficiency programs has proven much less expensive than producing $1 \mathrm{kWh}$ of energy by building a new power plant.

2. Reduced greenhouse gas emissions. Less energy production means less carbon dioxide emissions from power plants. This contributes to environmental benefits such as slowing down environmental pollution and global warming and preserving natural resources and the ecosystem.

3. Improved competitiveness for local manufacturers. Local companies that upgrade the efficiency of their products can compete better with multi-national companies, especially with lower production costs.

4. Higher consumer disposable income. Less spending on electric bills increases consumer purchasing power for other products, which helps local businesses.

5. Increased cash flow in the local economy. With higher disposable income, consumers are more willing to spend, thus, injecting money into the local economy.

6. Improved Trade Balance. Decrease in energy demand will reduce the consumption of indigenous resources (i.e. natural gas and oil), allowing more to be exported (for Lebanon, less to be imported). Increased export earnings (or less import spending) help alleviate trade deficit of Arabian countries.

7. Avoided future energy deficit as power demand rises. Energy exporting countries have become net importers due to dramatic increases in electricity demand. Energy-efficiency programs can help slow down the demand and prevent energy deficit in the future.

\section{Research and Training}

UTI was established via cooperation between the Housing and Building National Research Centre - HBRC in Cairo, and the Institute for Housing and Urban Development Studies - HIS in Rotterdam within the framework of the development cooperation between the Governments of the Netherlands and Egypt. Urban Training Institute UTI was officially founded in December 1997, with the technical and managerial assistance provided by the Institute for
Housing and Urban Development Studies - HIS from Rotterdam. The assistance provided by IHS focused first on capacity and institution building and human resources development which were required for UTI establishment. After its foundation; UTI started a new cooperation with another Dutch institute: Faculty of Geo-Information Science and Earth Observation (ITC). Through this cooperation, UTI received technical support in the field of Geographic Information System (GIS).

\subsection{UTI Mission and partnership}

To strengthen institutional and human resources capacities that can nurture the improvement of the living and environmental conditions of cities in Egypt and the Middle East. UTI organizes training programs in cooperation with many international organizations (Institute for Housing and Urban Development Studies (IHS), the Faculty of Geo-Information Science, Earth Observation (ITC) in the Netherlands and Ecopolis Europe institute. UTI also signed cooperation protocols, exchange programs and expertise, and training programs with a number of international bodies such as the German aid program (GIZ), and the United Nations human Settlements Program (UN-Habitat).

On the local level, UTI collaborates with local agencies and several national partners like, General Organization of Physical Planning (GOPP), Egyptian Environmental Affairs Agency (EEAA) subordinate to the Ministry of State for Environmental Affairs, Cairo University, Ain Shams University, and the Social Fund for Development (SFD). The Institute has been developing a strategy for the training year 2013 - 2014 that will suit all levels and targeted groups, by using new mechanisms. The training strategy for this year depends on covering the various aspects of sustainable urban development in order to achieve the message adopted by the Institute. These aspects comprise: environmental - economic - planning social aspects as well as the sustainable management of these aspects.

It has also been taken into consideration, the development of special programs for the preparation of decisionmakers and senior and middle levels that working in departments and ministries on the levels of general managers and managers of administrations and the relevant sections of urban development of the decisionmakers. The trainee is to be trained in one of the disciplines and modern and global trends that will help him-her to cope with the rapid urbanization processes taking place in the Arab world now, and teach him- her how to identify sustainable urban development policies that should be followed to address the problems of urban growth.

\subsection{Experience and Capabilities}

UTI has become training and capacity building institution specialized in post-graduate and tailor-made training, 
policy research and technical advisory services. It focuses on urban management \& planning, housing \& real estate development policies, informal settlements upgrading and urban renewal, urban environmental planning \& management, urban infrastructure project management and urban finance. UTI provides tailor-made and specialized training to professionals, technical cadre and policy makers working in central and local government agencies, NGO's, private sector and educational institutions.

The regional focus of UTI activities is based on the principle of comparative advantage and partnerships with local and regional institutions that help in building better cities and sustainable urban development in the Middle East. UTI tries to achieve this goal by combining institution building, specialized training, policy research and technical assistance to various sub-levels of government as well as to organizations from the private, community and education sectors. UTI three main streams of activities are Training, Research and Advisory Services as they are inter-connected. Beside academic research, UTI carries out a series of case-study and issue-focused research that aims at the development of training support materials based on Egyptian experiences. Research is geared to institutional analysis and the assessment of government policy effectiveness and implementation impacts, while the advisory services provide the practical experience, which adds to the solution-geared approach of the training.

\subsection{Training Strategy}

A broad training strategy is behind the philosophy of UTI. The primary objective is to enhance skill and effectiveness of individuals- professionals and practitioners from governmental and non-governmental organizations, and educational institutions - who are directly involved in planning, design, implementation and management of urban development programs and projects. At this stage UTI training focuses on individual skill enhancement. At later stage, institutional and organizational performance of governmental institutions is dealt with during the training programs. It becomes possible to address organizational performance and institutional responsiveness through the design of tailormade programs which address more effectively the needs of specific local organizations and their staff. This means in practice the linkage between training, on-the-job technical assistance and institutional- organizational restructuring. Only then, the impact of training on institutional reform and organizational performance can be properly assessed. From December 1997 till now, more than 7500 professionals (from different disciplines) have attended training programs at UTI. Preparing the strategic plans for villages as part of the national program to prepare strategic plans for the 4200 Egyptian villages through a participatory approach .UTI prepared 500 plans until now in several governorates Behira, Sharikya, Qalubia, Beni Swef and Fayoum governorates. UTI conducted a preliminary survey of environmental, urban, social, and economical problems in the villages of the governorates. The survey was designed to provide and assessment of prevailing problems conditions and practices in the villages of those selected centers, and provide a viable plan of action for critical environmental problems. The study focused on water, irrigation canals pollution, as well as solid waste and sludge management. Based on the results of the survey, UTI presented a set of guidelines for priority action, focusing on programming needs and requirements for immediate interventions.

Boulaq El- Dakrour participatory Upgrading Project: This Project is to develop a geographic information map for the small and medium scale enterprises in Boulaq ElDakrour. And to prepare an Action Plan for the improvement of El-Zomor road. Moreover, UTI is contracted by the GTZ participatory Urban Management program at the Ministry of planning to undertake several research and training activities.

\section{Holistic (Overall) Approach}

The assessment of the overall energy performance of a building, including the technical building systems, comprises a number of successive steps, which can be schematically visualized as a pyramid, figure 1 .

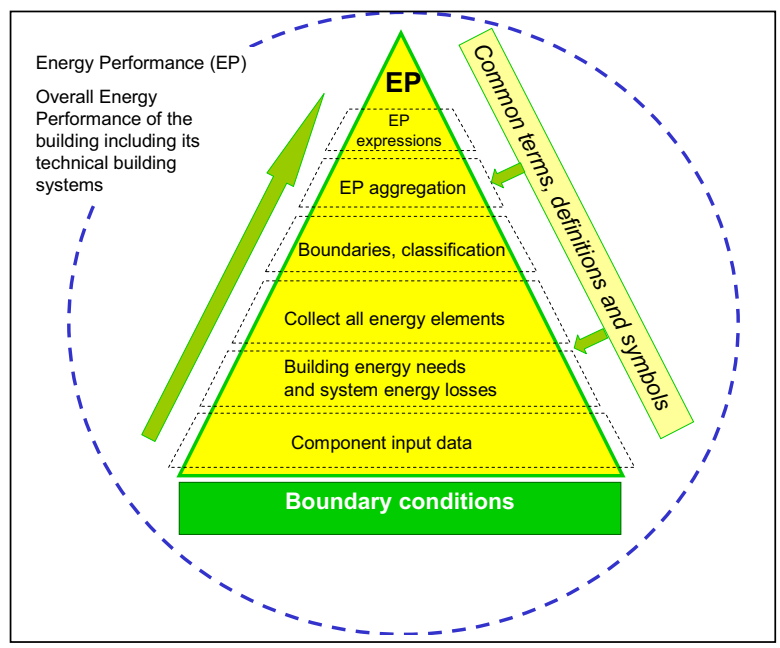

Fig. 1. Overall building energy performance, Ref.11

Sets of common terms, definitions and symbols are essential for all segments from top to bottom. These cover terms such as energy needs, technical building systems, auxiliary energy use, recoverable system losses, primary energy and renewable energy. 
The top segment of the pyramid is the main output: the energy performance and the energy performance certificate of the building.

The second segment provides the inputs for the top segment: one or more numerical indicators expressing the energy performance (such as overall energy use per square meter conditioned floor area, EP), a classification and ways to express the minimum energy performance requirements $\left(\mathrm{EP}_{\max }\right)$.

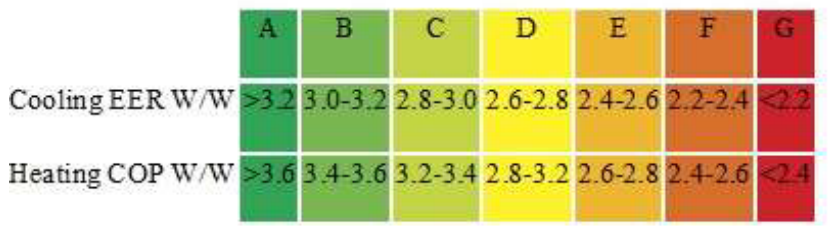

Fig. 2 . Example of energy Labeling certificate,Ref.9

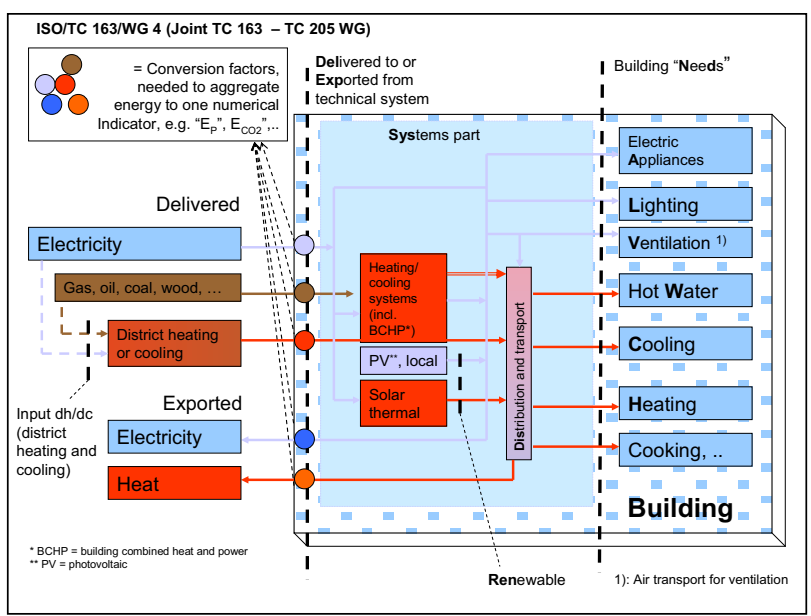

\section{Fig. 3. Boundaries and main building energy performance elements, Ref.11}

The third segment describes the principles and procedures on the weighting of different energy carriers (such as electricity, gas, oil or wood) when they are aggregated to overall amount of delivered (and exported) energy. For instance, this may be expressed as total primary energy $\left(E_{\mathrm{P}}\right)$ or carbon dioxide emission $\left(E_{\mathrm{CO} 2}\right)$.

The fourth segment specifies the categorization of building types (for example, office spaces, residential or retail) and specification of the boundaries of the building.

The fifth segment provides procedures on the breakdown of the building energy needs and system energy losses, aiming at gaining clear insights into where energy is used.
The sixth segment provides the building energy needs and energy use for each application (heating, cooling, etc.) and interactions between them.

The seventh segment provides the input data on components, such as thermal transmission properties, air infiltration, solar properties of windows, and energy performance of system components and efficiency of lighting. The standards that provide the procedures on boundary conditions comprise external climatic conditions, indoor environment conditions (thermal and visual comfort, indoor air quality, etc.), standard operating assumptions (occupation) and national legal restrictions.

\section{Energy Efficient Buildings}

\subsection{Energy declaration of Buildings}

The primary use of energy declarations is to:

1. Create consciousness of energy efficiency in buildings and also improve the knowledge of energy use in buildings;

2. Use the information to determine if the building works as well as possible with regard to its technical design;

3. Use the information for benchmarking;

4. Use the information for suggesting measures and recommendations for reducing the energy use;

5. Provide the information necessary to make calculations of the environmental impact due to the energy use, e.g. $\mathrm{CO} 2$ emission;

6. Describe selected energy properties of the building;

7. Give the basis for a common energy performance certification of a building

Depending on the purpose of the energy declaration, different procedures can be of interest. Different actors need different information. E.g. for benchmarking and for explaining the $\mathrm{CO} 2$ emission, it can be enough to read the total energy supply to the building and only adjust these figures to normal outdoor climate and to the heated area. For giving relevant advice to the property owner which measures are cost effective a very careful examination and calculation of the building's energy balance is necessary.

One way to proceed is to make the energy calculation in different steps for existing buildings. The first is to collect measured energy-use, e.g. from energy bills, and make a benchmarking to decide if the actual building is better or worse compared to similar buildings. If the energy-use seems to be higher than the average for a comparable grouping of buildings a second step is to make a careful energy calculation that can be compared to the measured energy use. This has to be done for identifying what kind of measures can be recommended in order to reduce the energy use in the building. For 
benchmarking, it can perhaps also be of interest to compare the measured energy use in the building examined with the estimated energy use in a building that is built with the best available technology. Alternatively, compared with a building that meets the requirement in the existing building codes. Some important aspects necessary to take into consideration when developing a common tool for energy declaration of buildings is discussed, $(12,13)$. The discussion is here focused on residential buildings but similar principles are relevant for other types of buildings.

\subsection{Energy Declaration of Existing Buildings}

For most existing buildings the energy use usually is well known via the energy bills. The construction details are on the other hand not very well documented. Calculations of the energy use will thus in most cases be very uncertain and difficulties will occur when giving relevant recommendations of cost-effective energy conservation measures.

The energy declaration needs a combination of tools for calculations based on the information from the energy bills. For existing buildings the energy declarations can be based on both measurements and calculations in order to fulfill the purposes mentioned above. Normally the heating bills are based on measured heat delivered to the building. In most cases, the energy for domestic hot water is included in the heating bill. To get the total energy use in a building, the electricity to run the building and household electricity have to be added. In electrically heated houses, common in e.g. Sweden, the house-owner just got one bill covering the total energy use. To make the measured values objective and comparable several corrections and calculations are necessary:

- It is necessary to check that the indoor thermal comfort and air quality meet agreed requirements

- Corrections of the heat use to normal outdoor climate primarily outdoor temperature (maybe also solar heat gain)

- Necessary corrections for internal heat gains, e.g. differences in household electricity

\subsection{Energy Declaration of New Buildings}

The energy declaration of a new building has to be based on calculations. In comparison with existing buildings, the knowledge of the building construction is very good. The most critical part for the outcome of calculations is the choice of input-data. To achieve good comparability, a common procedure to determine input data like energy for domestic hot water, household electricity or electricity for the activity in the building, choice of indoor temperature, electricity for operating the building, energy for lighting etc. has to be developed.

In many countries many new buildings have very low energy demand for heating. The solar heat gain, internal gains and energy losses from equipment etc. cover the major part of the heating demand. In several buildings the internal gains etc. are so large that cooling is necessary even in temperate climate. For buildings with large glassed areas and/or large internal gains the energy calculations need to be done on hourly basis. Many modern apartment blocks, offices, education buildings and restaurants need very little heat supply from the heating system and need very often air-conditioning to get an acceptable thermal comfort.

\subsection{Issues for International Collaboration}

- develop standardized tools for the calculation of the energy performance of buildings taking into account the aspects outlined in ISO 13790 that cover many aspects but still has to be completed.

- define system boundaries for the different building categories and different heating systems

- prepare models for expressing requirements on indoor air quality, thermal comfort in winter and when appropriate in summer, visual comfort, etc.

- develop transparent systems to determine necessary input data for the calculations, incl. default values on internal gains

- provide transparent information regarding output data (reference values, benchmarks, etc.)

- define comparable energy related key values $(\mathrm{kWh} / \mathrm{m} 2$, $\mathrm{kWh}$ per person, $\mathrm{kWh}$ per apartment, $\mathrm{kWh}$ per produced unit etc.) The areas/volumes need to be defined.

- develop a method to translate net energy, used in the building, to primary energy and $\mathrm{CO} 2$ emissions

- develop a common procedure for an "energy performance certificate"

- develop and compile relevant standards applicable for each individual building category.

An example of the energy efficiency indication in buildings is highlighted in ISO 23045.

\section{Summary of Conclusions}

It can be concluded that it is important to incorporate an energy performance directive as a Standard in our region such a goal will aid energy savings in large buildings and set regulations to energy efficient designs that are based on Standard calculation methods. It is recommended to:

1. Develop standardized tools for the calculation of the energy performance of buildings

2. Define system boundaries for the different building categories and different heating systems

3. Prepare models for expressing requirements on indoor air quality, thermal comfort in winter and when appropriate in summer, visual comfort, etc.

4. Define comparable energy related key values $(\mathrm{kWh} / \mathrm{m} 2, \mathrm{kWh}$ per person, $\mathrm{kWh}$ per apartment, $\mathrm{kWh}$ 
per produced unit etc.) and to develop a common procedure for an "energy performance certificate"

5. Design, construct and operate a solar decathlon (Building) that can meet the rural and desert requirements and save the diminishing fossil fuel sources.

\section{References}

1. Khalil, E.E. "Energy Performance of Buildings Directive in Egypt: A New Direction, HBRC Journal, Vol. 1, 2005.

2. Medhat, A.A. and Khalil, E.E. "Thermal Comfort Meets Human Acclimatization in Egypt" Proceeding of Healthy Building, Vol.2, pp25, June 2006.

3. Khalil,E.E. "Energy Performance Of Commercial Buildings In Egypt: A New Direction", Proceedings, Energy2030, Abu Dhabi, November 2006

4. Kosonen, R. "Displacement ventilation for room air moisture control in hot and humid climate", Roomvent 2002, pp. 241-244, 2002.

5. Leite, B.C.C. and Tribess, A. "Analysis of under floor air distribution system: Thermal comfort and energy consumption", Roomvent 2002, pp. 245-248, 2002.

6. Kameel, R., and Khalil, E. E., "Prediction of turbulence behavior using $\mathrm{k}-\varepsilon$ model in operating theatres", Roomvent 2002, pp. 73-76, 2002.

7. Khalil, E.E."Arab-Air Conditioning And Refrigeration Code For Energy-Efficient Buildings", Arab Construction World, Vol. 28, NO.8, pp.24-26, August 2008

8. Khalil, E.E. "Air Conditioning and Refrigeration Code For Energy-Efficient Buildings in the Arab World”, Journal of Kuwait Society of Engineers, Vol. 100, pp. 94-95, September 2008.

9. ISO publications ISO 23045_2009, January 2009.

10. Federal Register / Vol. 72 , No. 245 / Friday, December 21, 2007 / Rules and Regulations 72565.

11. Van Dijk , D. and Khalil, E.E., "Future cities Building on energy efficiency" ISO Focus, pp. 25-27, May 2011

12. Khalil,E.E. Holistic Approach to Smart Buildings from Construction Material to Services, OJEE, Open Journal of Energy Efficiency, Vol.1 No.3, pp.48-56, December 2012.

13. Khalil,E.E. Design of Energy Efficient Built Environment, CHRVI Middle East 2013 Seminar, Doha, Qatar, June 2013

14. Building Energy Efficiency in Cold Climates, Encyclopedia of Sustainable Technologies, 2017, Pages 149-157

15. IEA EBC annex 53: Total energy use in buildingsAnalysis and evaluation methods, Energy and Buildings, Volume 152, Page 124-136, 2017. 